\title{
The riesz convergence and riesz core of double sequences
}

\author{
Abdullah M Alotaibi ${ }^{*}$ and Celal Çakan ${ }^{2}$
}

* Correspondence: celal. cakan@inonu.edu.tr

${ }^{1}$ Department of Mathematics, King Abdulaziz University, P.O. Box 80203, Jeddah 21589, Saudi Arabia Full list of author information is available at the end of the article

\section{Abstract}

In this article, we have introduced the Riesz convergence and Riesz core of double sequences and determined the necessary and sufficient conditions on a fourdimensional matrix $A$ to yield $P_{R}-\operatorname{core}\{A x\} \subseteq P$ - core $\{x\}$ and $P_{R}$ - core $\{A x\} \subseteq s t_{2}$ - core $\{x\}$ for all $x \in \ell_{\infty}^{2}$.

Mathematics Subject Classification 2000: 40C05; 40J05; 46A45.

Keywords: double sequences, four dimensional matrices, Riesz mean, core theorems

\section{Introduction}

A double sequence $x=\left[x_{j k}\right]_{j, k=0}^{\infty}$ is said to be convergent in the Pringsheim sense or Pconvergent if for every $\varepsilon>0$ there exists an $N \in \mathbb{N}$ such that $\left|x_{j k}-\ell\right|<\varepsilon$ whenever $j, k$ $>N$, [1]. In this case, we write $P$ - $\lim x=\ell$. By $c_{2}$, we mean the space of all $P$-convergent sequences.

A double sequence $x$ is bounded if

$$
\|x\|=\sup _{j, k \geq 0}\left|x_{j k}\right|<\infty
$$

By $\ell_{\infty}^{2}$ we denote the space of all bounded double sequences.

Note that, in contrast to the case for single sequences, a convergent double sequence need not be bounded. So, we denote by $c_{2}^{\infty}$ the space of double sequences which are bounded and convergent.

Let $E \subseteq \mathbb{N} \times \mathbb{N}$ and $E(m, n)=\{(j, k): j \leq m, k \leq n\}$. Then, the double natural density of $E$ is defined by

$$
\delta_{2}(E)=P-\lim _{m, n} \frac{|E(m, n)|}{m n}
$$

if the limit on the right hand side exists; where the vertical bars denotes the cardinality of the set $E(m, n)$.

A real double sequence $x=\left[x_{j k}\right]$ is said to be statistical (or briefly st-) convergent [2] to the number $L$ if for every $\varepsilon>0$, the set $\left\{(j, k):\left|x_{j k}-L\right|>\varepsilon\right\}$ has double natural density zero. In this case, we write $\mathrm{st}_{2}-\lim x=L$. Let $s t_{2}$ be the space of all st-convergent double sequences. Clearly, a convergent double sequence is also st-conver-

(c) 2012 Alotaibi and Çakan; licensee Springer. This is an Open Access article distributed under the terms of the Creative Commons Attribution License (http://creativecommons.org/licenses/by/2.0), which permits unrestricted use, distribution, and reproduction in any medium, provided the original work is properly cited. 
gent but the converse it is not true, in general. Also, note that a st-convergent double sequence need not be bounded. For example, consider the sequence $x=\left[x_{j k}\right]$ defined by

$$
x_{j k}=\left\{\begin{array}{cc}
j k, & \text { if } j \text { and } k \text { are squares } \\
1, & \text { otherwise. }
\end{array}\right.
$$

Then, clearly $s t_{2}-\lim x=1$. Nevertheless $x$ neither convergent nor bounded. The $s t_{2}$ lim sup and $s t_{2}$ - lim inf of a double sequence were introduced in [3] and also the statistical core of a double sequence was defined by the closed interval $\left[s t_{2}-\lim\right.$ sup, $s t_{2}$ $\lim$ inf].

Let $A=\left[a_{j k}^{m n}\right]_{j, k=0}^{\infty}$ be a four-dimensional infinite matrix of real numbers for all $m, n=$ $0,1, \ldots$. The sums

$$
y_{m n}=\sum_{j=0}^{\infty} \sum_{k=0}^{\infty} a_{j k}^{m n} x_{j k}
$$

are called the $A$ - transforms of the double sequence $x=\left[x_{j k}\right]$. We say that a sequence $x=\left[x_{j k}\right]$ is $A$-summable to the limit $\ell$ if the $A$-transform of $x=\left[x_{j k}\right]$ exists for all $m, n$ $=0,1, \ldots$ and is convergent to $\ell$ in the Pringsheim sense, i.e.,

$$
\lim _{p, q \rightarrow \infty} \sum_{j=0}^{p} \sum_{k=0}^{q} a_{j k}^{m n} x_{j k}=y_{m n}
$$

and

$$
\lim _{m, n \rightarrow \infty} y_{m n}=\ell .
$$

We say that a matrix $A$ is bounded-regular if every bounded-convergent sequence $x$ is $A$-summable to the same limit and the $A$-means are also bounded. The necessary and sufficient conditions for $A$ to be bounded-regular or $R H$ - regular are known (see $[4,5])$.

A double sequence $x=\left[x_{j k}\right]$ of real numbers is said to be Cesáro convergent to a number $L$ if and only if there exists an $L \in \mathbb{R}$ such that

$$
\lim _{p, q \rightarrow \infty} \frac{1}{(p+1)(q+1)} \sum_{j=1}^{p} \sum_{k=1}^{q} x_{j k}^{m n}=L,
$$

and is denoted by $C_{1}-\lim x=L$. We denote the space of all Cesáro convergent double sequences by $C_{1}$. That is,

$$
C_{1}=\left\{x \in \ell_{\infty}^{2}: \exists L \in \mathbb{R}_{\ni} \quad C_{1}-\lim x=L\right\} .
$$

The concept core of single sequences (see [6]) was extended by Patterson [7] to the double sequences by defining the Pringsheim core (or $P$-core) of a real bounded double sequence $x=\left[x_{j k}\right]$ as the closed interval $[P-\lim \inf x, P-\lim \sup x]$. Later this concept has been studied by many authors. For example we refer $[2,8-10]$. 
Let

$$
C_{1}^{*}(x)=\limsup _{p, q \rightarrow \infty} \frac{1}{(p+1)(q+1)} \sum_{j=0}^{p} \sum_{k=0}^{q} x_{j k} .
$$

The Cesáro core (or $P_{C^{-}}$-core) of a real-valued bounded double sequence $x=\left[x_{j k}\right]$ has been defined by the closed interval $\left[-C_{1}^{*}(-x), C_{1}^{*}(x)\right]$ in [11]. Also; where an inequality related to the $P_{C}$ and $P$-cores has been investigated.

In this article we have introduced the Riesz convergence and Riesz core of a double sequence and also we have investigated some inequalities related to the $P_{-}$, statistical and Riesz cores.

\section{Main results}

Definition 2.1. Let $\left(q_{i}\right),\left(p_{j}\right)$ be sequences of non-negative numbers which are not all zero and $Q_{m}=q_{1}+q_{2}+\cdots+q_{m}, q_{1}>0, P_{n}=p_{1}+p_{2}+\cdots+p_{n}, p_{1}>0$. Then, the transformation given by

$$
t_{m n}^{q p}(x)=\frac{1}{Q_{m}} \frac{1}{P_{n}} \sum_{i=1}^{m} \sum_{j=1}^{n} q_{i} p_{j} x_{i j}
$$

is called the Riesz mean of double sequence $x=\left[x_{j k}\right]$.

Definition 2.2. If $P-\lim t_{m n}^{q p}(x)=s, s \in \mathbb{R}$, then the sequence $x=\left[x_{j k}\right]$ is said to be Riesz convergent to $s$.

If $x=\left[x_{j k}\right]$ is Riesz convergent to $s$, then we write $P_{R^{-}} \lim x=s$. In what follows $c_{R}^{2}$ will denote the set of all Riesz convergent sequences. Since a Riesz convergent double sequence need not be bounded, by $c_{R}^{2, \infty}$ we will denote the set of all bounded and Riesz convergent double sequences. $c_{0, R}^{2, \infty}$ will denote the set of all double sequences which bounded and Riesz convergent to zero.

Note that in the case $q_{i}=1$ for all $i$ and $p_{j}=1$ for all $j$, the Riesz mean reduced to the Cesáro mean and the Riesz convergence is said to be Cesáro convergence.

Now, we will give some lemmas characterized some classes of matrices related to the $c_{R}^{2, \infty}$.

Lemma 2.3. $\boldsymbol{A}$ matrix $A=\left(a_{j k}^{m n}\right) \in\left(\ell_{\infty}^{2}, c_{0, R}^{2, \infty}\right)$ if and only if

$$
\begin{aligned}
& \|A\|=\sup _{m n} \sum_{j k}\left|a_{j k}^{m n}\right|<\infty, \\
& P-\lim _{m n} \alpha(m, n, r, s, q, p)=0(r, s \in \mathbb{N}), \\
& P-\lim _{m n} \sum_{r}|\alpha(m, n, r, s, q, p)|=0(s \in \mathbb{N}), \\
& P-\lim _{m n} \sum_{s}|\alpha(m, n, r, s, q, p)|=0(r \in \mathbb{N}), \\
& P-\lim _{m n} \sum_{r s}|\alpha(m, n, r, s, q, p)|=0,
\end{aligned}
$$


where

$$
\alpha(m, n, r, s, q, p)=\frac{1}{Q_{r}} \frac{1}{P_{s}} \sum_{j=1}^{r} \sum_{k=1}^{s} q_{j} p_{k} a_{j k}^{m n}
$$

Proof. Let $A=\left(a_{j k}^{m n}\right) \in\left(\ell_{\infty}^{2}, c_{0, R}^{2, \infty}\right)$. This means that $A x$ exists for all $x=\left[x_{j k}\right] \in \ell_{\infty}^{2}$ and $A x \in c_{0, R}^{2, \infty}$ which implies (2.1). Let us define a sequence $y=\left[y_{r s}\right]$ by

$$
y_{r s}=\left\{\begin{array}{cc}
\operatorname{sgn} \alpha\left(m_{i}, n_{j}, r, s, q, p\right), & r_{i-1}<r<r_{i,} \quad s_{j-1}<s<s_{j} \\
0 & \text { otherwise. }
\end{array}\right.
$$

Then, the necessity of (2.5) follows from $P-\lim t_{r s}^{q p}(A x)$.

It is known by the assumption that

$$
P-\lim \sum_{r, s} \alpha(m, n, r, s, q, p) x_{j k}=0 .
$$

So; if we define the sequences $e_{i j}^{r s}, e^{r}, e^{s}$ as follows

$$
e_{i j}^{r s}= \begin{cases}1, & (j, k)=(r, s) \\ 0 & \text { otherwise }\end{cases}
$$

$e^{r}=\Sigma_{s} e^{r s}(s \in \mathbb{N})$ and $e^{s}=\Sigma_{r} e^{r s}(r \in \mathbb{N})$, then the necessity of (2.2), (2.3), and (2.4) follows from $P-\lim t_{r s}^{q p}\left(A e^{r s}\right), P-\lim t_{r s}^{q p}\left(A e^{r}\right)$ and $P-\lim t_{r s}^{q p}\left(A e^{s}\right)$, respectively.

Since the proof of the sufficiency part is routine, we omit the details.

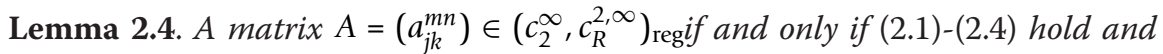

$$
P-\lim _{m n} \sum_{r s}|\alpha(m, n, r, s, q, p)|=1 .
$$

Proof. The necessity of the conditions can be shown by the same way used in the proof of Lemma 2.3.

For the sufficiency let the conditions hold and $x=\left[x_{j k}\right] \in c_{2}^{\infty}$ with $P-\lim x_{j k}=L$, (say). Then, there exists an $N>0$ such that $\left|x_{j k}\right|<|L|+\varepsilon$ for every whenever $j, k>N$. Now; let us write

$$
\begin{aligned}
\sum_{r s} \alpha(m, n, r, s, q, p) x_{r s}=\sum_{r=0}^{N} \sum_{s=0}^{N} \alpha(m, n, r, s, q, p) x_{r s} & +\sum_{r=N+1}^{\infty} \sum_{s=0}^{N-1} \alpha(m, n, r, s, q, p) x_{r s} \\
& +\sum_{r=0}^{N-1} \sum_{s=N+1}^{\infty} \alpha(m, n, r, s, q, p) x_{r s} \\
& +\sum_{r=N+1}^{\infty} \sum_{s=N+1}^{\infty} \alpha(m, n, r, s, q, p) x_{r s}
\end{aligned}
$$


which implies that

$$
\begin{aligned}
\left|\sum_{r s} \alpha(m, n, r, s, q, p) x_{r s}\right|=\|x\| \sum_{r=0}^{N} \sum_{s=0}^{N}|\alpha(m, n, r, s, q, p)| & +\|x\| \sum_{r=N+1}^{\infty} \sum_{s=0}^{N-1}|\alpha(m, n, r, s, q, p)| \\
& +\|x\| \sum_{r=0}^{N-1} \sum_{s=N+1}^{\infty}|\alpha(m, n, r, s, q, p)| \\
& +(|L|+\varepsilon)\left|\sum_{r=N+1}^{\infty} \sum_{s=N+1}^{\infty} \alpha(m, n, r, s, q, p)\right| .
\end{aligned}
$$

So, by letting $m, n \rightarrow \infty$ under the light of the assumption, we get that $P-\lim t_{r s}^{q p}(A x)=L$.

This completes the proof.

Lemma 2.5. A matrix $A=\left(a_{j k}^{m n}\right) \in\left(s t_{2} \cap \ell_{\infty}^{2}, c_{R}^{2, \infty}\right)_{\text {regif }}$ and only if

$$
\begin{aligned}
& A=\left(a_{j k}^{m n}\right) \in\left(c_{2}^{\infty}, c_{R}^{2,8}\right)_{\mathrm{reg}} \\
& P-\lim _{m n} \sum_{r, s \in E}|\alpha(m, n, r, s, q, p)|=0
\end{aligned}
$$

for every $E \subset \mathbb{N} \times \mathbb{N}$ with $\delta_{2}(E)=0$.

Proof. If $A=\left(a_{j k}^{m n}\right) \in\left(s t_{2} \cap \ell_{\infty}^{2}, c_{R}^{2, \infty}\right)_{\text {reg, }}$ the necessity of (2.7) follows from the fact that $c_{2}^{\infty} \subset s t_{2} \cap \ell_{\infty}^{2}$. For the necessity of the condition (2.8), let us choose a sequence $z$ $=\left[z_{r s}\right]$ by

$$
z_{r s}= \begin{cases}x_{r s}, & r, s \in E \\ 0, & \text { otherwise }\end{cases}
$$

where $x=\left[x_{r s}\right] \in \ell_{\infty}^{2}$ and $E \subset \mathbb{N} \times \mathbb{N}$ with $\delta_{2}(E)=0$. Then; it is easy to see that $s t_{2}$ $\lim z=0$ and

$$
t_{r s}^{q p}(A z)=\sum_{r, s \in E} \alpha(m, n, r, s, q, p) x_{r s} .
$$

So; a matrix $B=\left[b_{r s}^{m n}\right]$ defined by

$$
b_{r s}^{m n}=\left\{\begin{array}{cl}
\alpha(m, n, r, s, q, p), & r, s \in E \\
0, & \text { otherwise }
\end{array}\right.
$$

for every $q, p$ is in the class $\left(\ell_{\infty}^{2}, c_{0, R}^{2, \infty}\right)$. Therefore, the necessity of (2.8) follows from the condition (2.5) of Lemma 2.3.

For the converse take a sequence $x=\left[x_{r s}\right] \in s t_{2} \cap \ell_{\infty}^{2}$ with $s t_{2}-\lim x=l$. Then; it is known that $\delta_{2}=\delta_{2}\left(\left\{(r, s):\left|x_{r s}-l\right| \geq \varepsilon\right\}\right)=0$ and $\left|x_{r s}-l\right|<\varepsilon$ whenever $r, s \notin E$. Now, write

$$
\sum_{r, s} \alpha(m, n, r, s, q, p) x_{r s}=\sum_{r, s} \alpha(m, n, r, s, q, p)\left(x_{r s}-1\right)+l \sum_{r, s} \alpha(m, n, r, s, q, p) .
$$


The inequality

$$
\begin{aligned}
\left|\sum_{r, s} \alpha(m, n, r, s, q, p)\left(x_{r s}-l\right)\right| & =\left|\sum_{r, s \in E} \alpha(m, n, r, s, q, p)\left(x_{r s}-1\right)+\sum_{r, s \notin E} \alpha(m, n, r, s, q, p)\left(x_{r s}-1\right)\right| \\
& \leq\left\|x_{r s}-l\right\| \sum_{r, s \in E}|\alpha(m, n, r, s, q, p)|+\varepsilon\|A\|
\end{aligned}
$$

and condition (2.8) implies that

$$
P-\lim _{m n} \sum_{r, s} \alpha(m, n, r, s, q, p)\left(x_{r s}-l\right)=0 .
$$

So; by letting $m, n \rightarrow \infty$ in (2.9) we have $P-\lim t_{r s}^{q p}(A x)=l$ and this completes the proof.

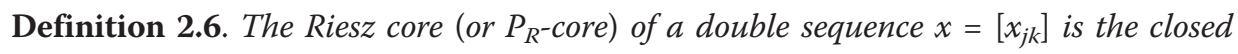
interval $\left[P-\lim \inf _{m, n} t_{m n}^{q p}(x), P-\lim \sup _{m, n} t_{m n}^{q p}(x)\right]$.

Note that in the case $q_{i}=1$ for all $i$ and $p_{j}=1$ for all $j$, Riesz core is reduced to the Cesáro core, [11].

Now; we are ready to give some inequalities related to the $P_{-}, P_{R^{-}}$and $s t_{2}$-core of double sequences.

Theorem 2.7. Let $\|A\|<\infty$. Then,

$$
P-\lim \sup _{r s}^{q p}(A x) \leq P-\lim \sup (x),
$$

for all $x \in \ell_{\infty}^{2}$ if and only if $A \in\left(c_{2}^{\infty}, c_{R}^{2, \infty}\right)_{\text {reg }}$ and

$$
P-\lim _{m, n} \sum_{r, s}|\alpha(m, n, r, s, q, p)|=1
$$

Proof. Let (2.10) holds for all $x \in \ell_{\infty}^{2}$. Then, it is easy to get that

$$
-P-\lim \sup (-x) \leq-P-\lim \sup t_{r s}^{q p}(-A x) \leq P-\lim \sup t_{r s}^{q p}(A x) \leq P-\lim \sup (x) .
$$

Since $-P-\lim \sup (-x)=P-\lim \inf (x)$ and $-P-\limsup t_{r s}^{q p}(-A x)=P-\liminf t_{r s}^{q p}(A x)$, by choosing $x \in c_{2}^{\infty}$, we reach that $P-\lim t_{r s}^{q p}(A x)=P-\lim (x)$. Since $x$ is arbitrary, this means that $A \in\left(c_{2}^{\infty}, c_{R}^{2, \infty}\right)_{\text {reg. }}$.

By Lemma 3.1 of Patterson [7], there exists a $y \in \ell_{\infty}^{2}$ with $\|y\| \leq 1$ such that

$$
P-\limsup t_{r s}^{q p}(A y)=P-\limsup \sum_{r, s}|\alpha(m, n, r, s, q, p)| .
$$

So; we have from assumption that

$$
P-\lim \sup \sum_{r, s}|\alpha(m, n, r, s, q, p)|=p-\lim \sup t_{r s}^{q p}(A y) \leq P-\lim \sup (y) \leq\|y\| \leq 1 .
$$

By the same way, one can see that

$$
P-\liminf \sum_{r, s}|\alpha(m, n, r, s, q, p)| \geq 1 .
$$

Therefore, by combining the inequalities (2.12) and (2.13), we obtain the necessity of (2.11). 
Conversely; suppose that $A \in\left(c_{2}^{\infty}, c_{R}^{2, \infty}\right)_{\text {reg }}$ and (2.11) holds. For any arbitrary bounded sequence $x=\left[x_{r s}\right]$, there exists $M, N>0$ such that $x_{r s} \leq P-\lim \sup x+\varepsilon$ whenever $r>M, s>N$. Now, we can write the following inequality,

$$
\begin{aligned}
\left|\sum_{r=0}^{\infty} \sum_{s=0}^{\infty} \alpha(m, n, r, s, q, p) x_{r s}\right| & =\mid \sum_{r=0}^{\infty} \sum_{s=0}^{\infty}\left(\frac{|\alpha(m, n, r, s, q, p)|+\alpha(m, n, r, s, q, p)}{2}\right. \\
& \left.-\frac{|\alpha(m, n, r, s, q, p)|-\alpha(m, n, r, s, q, p)}{2}\right) x_{r s} \mid \\
& \leq \sum_{r=0}^{\infty} \sum_{s=0}^{\infty}|\alpha(m, n, r, s, q, p)|\left|x_{r s}\right| \\
& +\sum_{r=0}^{\infty} \sum_{s=0}^{\infty}\left|(|\alpha(m, n, r, s, q, p)|-\alpha(m, n, r, s, q, p)) x_{r s}\right| \\
& \leq\|x\| \sum_{r=0}^{M} \sum_{s=0}^{N}|\alpha(m, n, r, s, q, p)| \\
& +\|x\| \sum_{r=M+1}^{\infty} \sum_{s=0}^{N}|\alpha(m, n, r, s, q, p)| \\
& +\|x\| \sum_{r=0}^{\infty} \sum_{s=N+1}^{N}|\alpha(m, n, r, s, q, p)| \\
& +(P-\lim \sup x+\varepsilon) \sum_{r=M+1}^{\infty} \sum_{s=N+1}^{\infty}|\alpha(m, n, r, s, q, p)| \\
& +\|x\| \sum_{r=0}^{\infty} \sum_{s=0}^{\infty}(|\alpha(m, n, r, s, q, p)|-\alpha(m, n, r, s q, p)) .
\end{aligned}
$$

Using the conditions characterized the class $\left(c_{2}^{\infty}, c_{R}^{2, \infty}\right)_{\text {reg }}$ and $(2.11)$, we reach that $P-\lim \sup t_{r s}^{q p}(A x) \leq P-\lim \sup (x)$ and this completes the proof of the theorem.

Theorem 2.8. Let $\|A\|<\infty$. Then,

$$
P-\lim \sup t_{r s}^{q p}(A x) \leq s t_{2}-\lim \sup (x),
$$

for all $x \in x \in \ell_{\infty}^{2}$ if and only if $A \in\left(s t_{2} \cap \ell_{\infty}^{2}, c_{R}^{2, \infty}\right)_{\mathrm{reg}}$ and (2.11) holds.

Proof. Let (2.14) holds for all $x \in \ell_{\infty}^{2}$. Then, by the same argument used in Theorem 2.7, one can see that $A \in\left(s t_{2} \cap \ell_{\infty}^{2}, c_{R}^{2, \infty}\right)_{\text {reg. }}$ On the other since $s t_{2}-\lim \sup (x) \leq P$ $\lim \sup (x)$ for all $x \in \ell_{\infty}^{2}$, the necessity of (2.11) follows from Theorem 2.7.

For the converse suppose that $A \in\left(s t_{2} \cap \ell_{\infty}^{2}, c_{R}^{2, \infty}\right)_{\text {reg }}$ and (2.11) holds. If $x=\left[x_{r s}\right] \in \ell_{\infty \prime \prime}^{2}$ it is known that for every $\varepsilon>0$,

$$
\delta_{2}(E)=\delta_{2}\left(\left\{(r, s): x_{r s}>s t_{2}-\lim \sup (x)+\varepsilon\right\}\right)=0
$$

and $x_{r s} \leq s t_{2}-\lim \sup (x)+\varepsilon$ whenever $r, s \notin E$. Taking this knowledge in the mind, let us write 


$$
\begin{aligned}
\sum_{r, s} \alpha(m, n, r, s, q, p) x_{r s} & \leq \mid \sum_{r, s} \frac{\left|\alpha(m, n, r, s, q, p) x_{r s}\right|+\alpha(m, n, r, s, q, p) x_{r s}}{2} \\
& +\sum_{r, s} \frac{\left|\alpha(m, n, r, s, q, p) x_{r s}\right|-\alpha(m, n, r, s, q, p) x_{r s} \mid}{2} \mid \\
& \leq\left|\sum_{r, s \in E} \alpha(m, n, r, s, q, p) x_{r s}+\sum_{r, s \notin E} \alpha(m, n, r, s, q, p) x_{r s}\right| \\
& +\|x\| \sum_{r, s}(|\alpha(m, n, r, s, q, p)|-\alpha(m, n, r, s, q, p)) \\
& \leq\|x\| \sum_{r, s \in E}|\alpha(m, n, r, s, q, p)| \\
& +\left(s t_{2}-\lim \sup (x)+\varepsilon\right) \sum_{r, s \notin E} \mid \alpha(m, n, r, s, q, p) \\
& +\|x\| \sum_{r, s}(|\alpha(m, n, r, s, q, p)|-\alpha(m, n, r, s, q, p)) .
\end{aligned}
$$

So, the conditions characterized the class $\left(s t_{2} \cap \ell_{\infty}^{2}, c_{R}^{2, \infty}\right)_{\text {reg }}$ and (2.11) imply that $P-\lim \sup t_{r s}^{q p}(A x) \leq s t_{2}-\lim \sup (x)+\varepsilon$. Since $\varepsilon$ was arbitrary, this completes the proof.

\section{Author details}

${ }^{1}$ Department of Mathematics, King Abdulaziz University, P.O. Box 80203, Jeddah 21589, Saudi Arabia ${ }^{2}$ Faculty of Education, Inönü University, 44280-Malatya, Turkey

\section{Authors' contributions}

AMA designed the problems and carried out the proof of the Lemmas. Ç̧ defined the Riesz core and gave the proof of the theorems. All authors read and approved the final manuscript.

\section{Competing interests}

The authors declare that they have no competing interests.

Received: 1 November 2011 Accepted: 7 March 2012 Published: 7 March 2012

\section{References}

1. Pringsheim, A: Zur theorie der zweifach unendlichen Zahlenfolgen. Math Ann. 53, 289-321 (1900). doi:10.1007/ BF01448977

2. Mursaleen, M, Edely, OHH: Statistical convergence of double sequences. J Math Anal Appl. 288, 223-231 (2003). doi:10.1016/j.jmaa.2003.08.004

3. Çakan, C, Altay, B: Statistically boundedness and statistical core of double sequences. J Math Anal Appl. 317(2):690-697 (2006). doi:10.1016/j.jmaa.2005.06.006

4. Hamilton, HJ: Transformations of multiple sequences. Duke Math J. 2, 29-60 (1936). doi:10.1215/S0012-7094-36-00204-1

5. Robison, GM: Divergent double sequences and series. Trans Am Math Soc. 28, 50-73 (1926). doi:10.1090/S0002-99471926-1501332-5

6. Knopp, K: Zur Theorie der Limitierungsverfahren (erste Mitteilung). Math Z. 31, 115-127 (1930)

7. Patterson, RF: Double sequence core theorems. Int J Math Math Sci. 22, 785-793 (1999). doi:10.1155/ S0161171299227858

8. Mursaleen, M: Almost strongly regular matrices and a core theorem for double sequences. J Math Anal Appl. 293 523-531 (2004). doi:10.1016/j.jmaa.2004.01.014

9. Mursaleen, $\mathrm{M}$, Edely, $\mathrm{OHH}$ : Almost convergence and a core theorem for double sequences. J Math Anal Appl. 293, 532-540 (2004). doi:10.1016/j.jmaa.2004.01.015

10. Mursaleen, M, Cakan, C, Mohiuddine, SA, Savas, E: Generalized convergence and statistical core of double sequences. Acta Math Sinica. 26(11):2131-2144 (2010). doi:10.1007/s10114-010-9050-2

11. Kayaduman, K, ïjakan, C: The Cesáro core of double sequences. Abstr Appl Anal 2011, 9 (2011). Article ID 950364

doi:10.1186/1029-242X-2012-56

Cite this article as: Alotaibi and Çakan: The riesz convergence and riesz core of double sequences. Journal of Inequalities and Applications 2012 2012:56. 